\title{
Examining family pre-pandemic influences on adolescent psychosocial wellbeing during the COVID-19 pandemic
}

\author{
Rosa S. Wong ${ }^{1,4} \cdot$ Keith T. S. Tung ${ }^{1} \cdot$ Xue Li $^{3,4} \cdot$ Celine S. Chui ${ }^{4} \cdot$ Winnie W. Y. Tso ${ }^{1,5} \cdot$ Tatia M. C. Lee $^{5,6} \cdot$ Ko Ling Chan $^{7}$. \\ Yun K. Wing ${ }^{2} \cdot$ King Wa Fu ${ }^{8} \cdot$ lan C. K. Wong ${ }^{4,9} \cdot$ Patrick Ip ${ }^{1}$
}

Accepted: 16 January 2022

(c) The Author(s), under exclusive licence to Springer Science+Business Media, LLC, part of Springer Nature 2022

\begin{abstract}
During the COVID-19 lockdown, with social distancing measures in place and a decrease in social activities, emotional states are more likely to be transferred between family members via increased interactions and communication. However, longitudinal evidence, particularly for early adolescents, is lacking. This study investigated family pre-pandemic influences on parental stress and adolescent psychosocial wellbeing during the COVID-19 pandemic. Data were collected from 233 adolescents and their parents before and during the initial phase of the pandemic. Parents reported their own stress level and perception of adolescent adjustment problems, whereas adolescents reported their own psychological distress level. In addition, adolescents also reported their satisfaction with family life in the pre-pandemic survey. Cross-lagged path models indicated reciprocal associations between parental stress and perception of adolescent adjustment problems. Compared to adolescents low in pre-pandemic family life satisfaction, those adolescents with higher levels of family life satisfaction before the pandemic reported lower levels of anxiety and stress only when parental stress showed no increase during the pandemic. Findings provide support for the mutual influences between parental stress and perceived adolescent adjustment problems during the pandemic. Special attention should be paid to those adolescents who undergo significant family life changes during the pandemic.
\end{abstract}

Keywords COVID-19 pandemic $\cdot$ Adolescent adjustment problems $\cdot$ Adolescent psychological distress $\cdot$ Parental stress Family satisfaction

\section{Introduction}

The 2019 novel coronavirus disease (COVID-19) has rapidly spread across the world since late 2019. Many countries had implemented either a full or partial lockdown to

Patrick lp

patricip@hku.hk

Ian C. K. Wong

wongick@hku.hk

1 Department of Paediatrics and Adolescent Medicine, The University of Hong Kong, Hong Kong SAR, China

2 Department of Psychiatry, Faculty of Medicine, The Chinese University of Hong Kong, Hong Kong SAR, China

3 Department of Medicine, The University of Hong Kong, Hong Kong SAR, China

4 Centre for Safe Medication Practice and Research, Department of Pharmacology and Pharmacy, The University of Hong Kong, Hong Kong SAR, China control the spread of COVID-19. The closure of schools, educational institutes, and activity areas has negatively affected over $91 \%$ of the world's student population (Lee, 2020). With a large proportion of people being confined indoors for a long period of time, there has been increasing

5 State Key Laboratory of Brain and Cognitive Sciences, The University of Hong Kong, Hong Kong SAR, China

6 Laboratory of Neuropsychology, The University of Hong Kong, Hong Kong SAR, China

7 Department of Applied Social Sciences, The Hong Kong Polytechnic University, Hong Kong SAR, China

8 Journalism and Media Studies Centre, The University of Hong Kong, Pokfulam, Hong Kong SAR, China

9 Research Department of Practice and Policy, UCL School of Pharmacy, London, UK 
concerns and attention to the mental health impact of school closures and other physical distancing requirements (Holmes et al., 2020; Van Lancker \& Parolin, 2020). Yet, the majority of research has focused on the prevalence and severity of mental health problems during lockdown. While a theoretical conception of family and child experiences during the current pandemic is emerging (Benner \& Mistry, 2020; Prime et al., 2020), there remains little empirical work to investigate the mechanisms underpinning these experiences.

During lockdown, with social distancing measures in place and a decrease in social activities, spending more time at home gives both opportunities and challenges that could affect family wellbeing. Findings of emotional changes in parents and children during the pandemic have been mixed. On one hand, the disruptions caused by the pandemic to employment, education, and socialization can intensify emotional disturbance in parents and children. It has been reported that increased caregiving burden during the pandemic is linked with parental reports of mental ill-health and perception of children's stress (Romero et al., 2020). On the other hand, for some families, the COVID-19 crisis can provide a unique opportunity to reconnect, create memories, and evaluate priorities. In a qualitative study of families in Australia, Evans et al. (2020) found that while most families reported mental health difficulties and strained relationships during the COVID-19 pandemic, some families were unaffected because they could cope with pandemic-related stressors using constructive communication and conflict resolution strategies.

Despite emerging evidence of a link between parenting stress and child outcomes during the COVID-19 pandemic, the magnitude of psychological and behavioural responses towards stressful societal conditions could vary from person to person, depending on the availability of personal and social resources before the event occurs (Lau et al., 2006; Ni et al., 2020). An investigation of the characteristics of family environment before the pandemic would be useful for improving our understanding of why and how mental health states in parents and children can change over the pandemic course. While research targeting young children is emerging (Liu et al., 2020; Xie et al., 2020), very few work has been done to clarify the mental health impact of COVID-19 pandemic on children reaching early adolescence and their families. Early adolescence is of special interest because this is a time of transition from childhood to adolescence, during which individuals may have greater vulnerability to mental illnesses particularly at difficult times such as COVID-19 (Benner \& Mistry, 2020).

\section{Family burden and stress during the COVID-19 pandemic}

As highlighted by $\mathrm{Wu}$ and $\mathrm{Xu}$ (2020), families face various challenges and stressors during the pandemic, including physical health and mental health concerns, economic stress, challenges in home-schooling, marital conflicts and intimate partner violence, and intensified parent-child dyadic tension, all of which can exacerbate psychological distress within the affected individuals in the absence of internal (e.g. personal traits and resilience) and external (e.g. emotional and financial support) coping resources. Although disadvantaged families are more likely to have insufficient resources, increased financial pressure due to unexpected events such as unemployment, income decline, and unmanageable debts during the pandemic can affect all families across socioeconomic strata (Fegert et al., 2020). It is thus important to recognize that the pandemic is causing unprecedented challenges to all families regardless of their socioeconomic background.

\section{Theoretical perspectives on parental stress and adolescent adjustment problems}

Different theoretical models have been suggested to explain how social disruption may impact child adjustment during the COVID-19 pandemic (Prime et al., 2020; Wu \& Xu, 2020). The family stress model, for example, suggests that stressors could strain caregivers' wellbeing and adaptive parenting behaviours, in turn leading to increased psychosocial difficulties in children (Conger et al., 2002). In particular, children's socio-emotional problems would likely arise when parents frequently express negative affect or unsupportive reactions to children's emotions (Breaux et al., 2016). As indicated by the family system theory (Schermerhorn \& Mark Cummings, 2008), family is an emotional unit where members could interact and exert influences on each other's thinking and behavior. Evidence suggests that emotions in one family member can spill over to the other members (Nelson et al., 2009). Although the parent-driven models are evident in the COVID-19 setting, very little attention has been paid to the impact of children's behaviour on parental mental health during the COVID-19 period. Notably, early adolescents just start to explore ways of becoming independent but still seek to maintain close relationships with family members (Lam et al., 2012). This independence-dependence struggle may make early adolescents more difficult to adjust to the challenges posed by the COVID-19 pandemic, which in turn could increase parental stress and affect how they perceive children's adjustment problems during this period. 


\section{Adolescent satisfaction with family life and mental health during the pandemic}

Family satisfaction refers to the positive affective state resulting from one's assessment of family aspects of his or her life in general (Pattusamy \& Jacob, 2017). Prime et al. (2020) suggested that pre-existing vulnerabilities within families could predispose children and families to pandemic-related stress and symptoms of anxiety and depression, whereas intact or strong family bond can protect individuals against these negative emotional states. A previous study examining the impact of $9 / 11$ attacks on children's post-traumatic stress (PTS) symptoms found that maternal acceptance pre-9/11 and helpful mother-child discussions about the event post-9/11 were linked with fewer PTS symptoms in children (Wilson et al., 2010). Research also found an association between increased family support and a lower risk of probable depression during social movements (Ni et al., 2020). In addition, youth who were social distancing because of parental rules reported greater belongingness during the initial phase of the COVID-19 pandemic (Oosterhoff et al., 2020). However, it is unclear whether such association still holds true for those adolescents who have been living in a conflictual and unsupportive family environment before the pandemic.

\section{The present study}

In Hong Kong, about $92 \%$ of the population are Chinese, and the remaining $8 \%$ are composed of various ethnic minorities (HKSAR Census \& Statistics Department, 2016). As a melting pot of eastern and western cultures, Hong Kong has its own social norms and practices, implementing localized containment strategies such as school closures for preventing the spread of COVID-19. In addition, Hong Kong is one of the most densely populated areas in the world and known for small space living. Prolonged confinement may heighten daily stress and other negative feelings that take a toll on mental and social well-being. (Tso et al., 2020), but longitudinal evidence examining family dynamics during the pandemic remains limited. To address these knowledge gaps, this study would have two parts. The first part was to examine the bidirectional relationship between parental stress and their perception of adolescent adjustment problems before and during the COVID-19 pandemic. The second part was to investigate whether adolescents' pre-pandemic satisfaction with family life would moderate the association of withinperson changes in 10 and 12 during the pandemic.

\section{Method}

\section{Participants}

Two-wave longitudinal data were obtained from a small subset of participants $(n=233)$ in the Healthy Kids cohort. Healthy Kids is a prospective longitudinal cohort study aiming to examine the effect of early-life socioeconomic status on long-term health and well-being (Wong et al., 2021). The pre-pandemic data were collected during April to August 2019 (T1), whereas the follow-up data were collected in late February 2020 (T2) when Hong Kong braced itself for the first outbreak of COVID-19 and suspended schools to contain the spread. The sample size was estimated based on the finding of a previous study reporting an effect size of $\beta=0.50$ for the association between parental stress and adjustment problems in children aged 2 to 12 years (Tso et al., 2020). Assuming a smaller effect size of $\beta=0.25$ and adjusting for up to ten confounders, at least 120 participants would be needed to detect such effect at 0.05 significance level and $80 \%$ statistical power.

\section{Procedure}

During T1, we approached and invited families in the Healthy Kids study to provide written consent for participation and complete the questionnaire survey. Based on their survey responses, we invited the schools where the cohort children were attending to help recruit other eligible families. With the permission from school principals, an invitation letter describing the study objectives and questionnaires was distributed to parents of all students in the target grade of the participating schools. Upon completion, participants who were interested to join returned their written informed consent and completed questionnaires by mail. At T2, due to school closure and other social distancing measures, parents and their adolescent children received a text message with a Web link to their respective online survey that contained the same questionnaires administered at T1. Data were subsequently exported by the research team for further analyses. The study was approved by the Institutional Review Board of the University of Hong Kong/Hospital Authority Hong Kong West Cluster (UW 18-057).

\section{Measures}

\section{Parental stress}

Parents completed a Chinese version of the Parental Distress subscale of the Parenting Stress Index - Short Form (PSI-SF-PD) (Abidin, 1997; Tam et al., 1994) at T1 and 
T2. The subscale consists of 12 items (e.g., "Feel trapped by parenting responsibilities") on a 5-point scale ranging from $1=$ extremely disagree to $5=$ extremely agree. The total score of the items reflects the parent's personal adjustment to parenthood, with higher scores indicating higher levels of stress associated with the care of the adolescent child. This scale showed good reliability with Cronbach's alpha of $\alpha=0.89$ at $\mathrm{T} 1$ and $\alpha=0.89$ at $\mathrm{T} 2$.

\section{Parental perception of adolescent adjustment problems}

Parents completed a Chinese version of the Strength and Difficulties Questionnaire (SDQ) (Goodman, 2001) at $\mathrm{T} 1$ and $\mathrm{T} 2$. The measure, which has demonstrated good psychometric properties in previous Chinese adolescent research (Huang et al., 2016; Liang et al., 2019), contains subscales for emotion problems (e.g., "Many fears, easily scared"), conduct problems (e.g., "Often fights with other children"), hyperactivity (e.g., "Restless, overactive, cannot stay still for long"), and peer problems (e.g., "Has at least one good friend"), and each subscale has five items, on a 3 -point scale ranging from $0=$ not true to $2=$ certainly true. According to the official scoring guidelines, there four problem behavior subscale scores can be combined to generate a total adjustment problem score, with higher scores indicating more adjustment problems. The Cronbach's alpha of this 20 -item measure was 0.83 at $\mathrm{T} 1$ and 0.79 at $\mathrm{T} 2$.

\section{Adolescent psychological distress}

Adolescents completed a Chinese version of the Depression Anxiety Stress Scale - 21 (DASS-21) (Taouk et al., 2001) at $\mathrm{T} 1$ and $\mathrm{T} 2$. The measure contains three scales for depression, anxiety, and stress, respectively, with seven items for each scale rated on a 4-point scale ranging from $0=$ did not apply at all to $3=$ applied very much or most of the time. Each scale has its own total score which is the sum of corresponding items multiplied by two, with higher scores indicating more symptoms. Cronbach's alphas in the current sample indicated good internal consistency for all the DASS-21 scales (Cronbach's alpha for Depression scale was 0.77 at $\mathrm{T} 1$ and 0.82 at T2; for Anxiety scale was 0.68 at T1 and 0.73 at $\mathrm{T} 2$; for Stress scale was 0.76 at $\mathrm{T} 1$ and 0.78 at $\mathrm{T} 2$ ).

\section{Adolescent pre-pandemic satisfaction with family life}

At T1, adolescents reported their level of satisfaction with family life by completing an adapted version of a family connectedness questionnaire (Resnick et al., 1997). The measure consists of four items (e.g., "Your mum is warm and loving toward you," "You are satisfied with relationship with mum," "Your dad is warm and loving towards you," "You are satisfied with relationship with dad") concerning specific aspects of family life. Adolescents were asked to indicate how much they agreed with each of the items on a 5-point scale ranging from $1=$ totally disagree to $5=$ totally agree The total score of the items was calculated, with higher scores indicating higher levels of family life satisfaction. To compare psychological distress levels between family life satisfaction subgroups, adolescents were classified as high and low levels using the median-split approach by a cut-off score of 18. The Cronbach's alpha of this measure was 0.93 .

\section{Covariates}

Informed by prior research (Elgar et al., 2013; Rothbaum \& Weisz, 1994; Wong et al., 2021), adolescent's age, gender, externalizing problems, frequency of dinner with parents, family socioeconomic status (SES), status of receiving financial assistance from the government, and parental marital status at T1 were used as covariates. Age and gender were recorded at enrolment. Parents self-reported their frequency of dinner with adolescent children and marital status. Adolescents' externalizing problems were rated by their parents using the SDQ externalizing scales (conduct problems and hyperactivity) (Goodman, 2001). In addition to the status of receiving financial assistance from the government, parents also provided information on their own education level, current employment status, and monthly household income which were z-scored and combined to form a family SES composite score using the principal component analysis with varimax rotation (Vyas \& Kumaranayake, 2006).

\section{Data analysis}

Pearson correlations were computed to examine the interrelationships between study variables. Prior to analysis, all variables were inspected for outliers and normality; all assumptions of multiple linear regression, including normality, homoscedasticity, and absence of multicollinearity were met. To investigate the stability and cross-lagged effects, we employed structural equational modelling (SEM) with full information maximum likelihood techniques using the multiple imputation by chained equations (MICE) R package (Buuren \& Groothuis-Oudshoorn, 2010). This two-wave autoregressive cross-lagged design allows for examination of influences over time of one construct on another and vice versa, while simultaneously controlling for stability as well as for associations between the two investigated constructs over time (Kline, 2015; Little et al., 2007). Specifically, we built one single model to include 
all autoregressive, correlated, and cross-lagged relationships with adjustment for several confounding effects including age, gender, and family SES. The autoregressive relationships accounted for stability over time for both the PSI-SF-PD sum score and the SDQ sum score. Additionally, we included the correlations between the sum score of PSI-SF-PD and that of SDQ at T1 and T2 to account for the strong association between these constructs (Barroso et al., 2018; Neece et al., 2012). Hence, the crosslagged effects can be interpreted as the predictive effects of (1) the sum score of PSI-SF-PD at T1 on that of SDQ at T2 and (2) the sum score of SDQ at T1 on that of PSISF-PD at T2. The goodness-of-fit of the estimated model was evaluated using four indicators: comparative fit index (CFI), Tucker Lewis Index (TLI), root mean square error of approximation (RMSEA), and standardized root mean square residual (SRMR). Good model fit is indicated by $\mathrm{CFI}>0.95$, TLI $>0.95$, RMSEA $<0.06$, and $\mathrm{SRMR}<0.08$ (Hu \& Bentler, 1999).

Next, we used hierarchical linear modelling (HLM) to examine the potential moderating effect of adolescent prepandemic family life satisfaction on the relationship between parental stress changes and adolescent psychological distress at T2. First, we tested the main effect of increased parental stress and adolescent pre-pandemic family life satisfaction on adolescent changes in psychological distress at T2. Second, we examined the effect of the interaction term of adolescent pre-pandemic family life satisfaction and increased parental stress on adolescent changes in psychological distress at T2. Age, gender, family SES, parental marital status, status of receiving financial assistance from the government, frequency of dinner with parents, and initial level of externalizing problems and distress variable of interest were entered as covariates into these analyses. Given missing values below $5 \%$ of the total number of values, we replaced the missing data of predictors and control variables using the multiple imputation by chained equations (MICE) R package (Buuren \& Groothuis-Oudshoorn, 2010) to include all cases $(n=233)$.

All data analyses were carried out using $\mathrm{R}$ version 3.6.3 (R Core Team, 2013). Standardized coefficients were reported, and p-values less than 0.05 were considered statistically significant in this study.

\section{Results}

\section{Descriptive statistics}

Table 1 displays the socio-demographic characteristics of the entire sample. There were no dropouts and reports of major adverse events or changes occurring in the parents or adolescents during the follow-up period, and none had been diagnosed with COVID-19 at the time of follow-up survey. Over $97 \%$ of the parent respondents were mothers. The mean age of the adolescents ( 92 males and 141 females) was 12.20 years $(\mathrm{SD}=0.43)$ at $\mathrm{T} 1.94 .4 \%$ of the parents were married and living together. Two families reported receiving government subsidies at T1. $138(59.2 \%)$ parents reported an increase in parental stress at $\mathrm{T} 2.93(40.2 \%)$ adolescents were perceived by parents to have an increase in adjustment problems at T2. $41(17.6 \%)$ adolescents experienced an increase in all three aspects of psychological distress (stress, depression, and anxiety) at T2. Specifically, 96 (41.9\%) reported an increase in depression levels, whereas $98(43.4 \%)$ and 91 (39.1\%) reported an increase in stress and anxiety levels, respectively. Intercorrelations among $\mathrm{T} 2$ emotional changes and $\mathrm{T} 1$ family life satisfaction are presented in Table 2. Adolescent T1 family life satisfaction correlated positively and significantly with their change in adjustment problems at $\mathrm{T} 2(\mathrm{r}=0.137, \mathrm{p}=0.038)$, suggesting that adolescents high in family life satisfaction before COVID-19 were more sensitive to the psychosocial impact of the outbreak.

\section{Part I-Cross-lagged analyses}

The bidirectional model, adjusting for age, gender, and family SES (Fig. 1), fit the data adequately, CFI $=0.973$; $\mathrm{TLI}=0.918 ; \mathrm{RMSEA}=0.082 ; \mathrm{SRMR}=0.064$. When parents perceived their adolescents to have more adjustment problems at $\mathrm{T} 1$, they would report higher stress levels at $\mathrm{T} 2(\beta=0.13$, $\mathrm{p}<0.05)$. Likewise, when parents reported higher stress levels at $\mathrm{T} 1$, they would perceive more adjustment problems in their adolescents at T2 $(\beta=0.23, \mathrm{p}<0.001)$. As for the autoregressive relationships, perception of adolescent adjustment problems at $\mathrm{T} 1$ and $\mathrm{T} 2$ were significantly associated with each other $(\beta=0.58, p<0.001)$. Similarly, parental T1 stress was also predictive of parental T2 stress $(\beta=0.58, \mathrm{p}<0.001)$.

\section{Part II-Moderation analyses}

Hierarchical regression analysis was used to examine the moderating effect of adolescent $\mathrm{T} 1$ family life satisfaction on the association of changes in parental stress and adolescent psychological distress at T2 (Table 3). The first step in the analysis examined whether T1 family life satisfaction would be associated with adolescent $\mathrm{T} 2$ emotional adjustment as well as whether parental stress would change with adolescent psychological distress over the study period. Results showed no significant associations between these variables after controlling for the effects of age, gender, family SES, parental marital status, status of receiving financial assistance from the government, frequency of dinner 
Table 1 Subject characteristics at $\mathrm{T} 1(\mathrm{n}=233)$

\begin{tabular}{ll}
\hline Study variables & \\
\hline Age, mean(SD) & $12.20(0.43)$ \\
Gender, n(\%) & $92(39.5)$ \\
Male & $141(60.5)$ \\
Female & $0.07(1.38)$ \\
Family socioeconomic status index, mean(SD) & $58,927(39,087)$ \\
Monthly household income (HKD), mean(SD) & $2(0.9)$ \\
Receiving financial assistance from the government, n(\%) & \\
Paternal educational level, n(\%) & $93(39.9)$ \\
Bachelor degree or above & $90(38.6)$ \\
Grade 10 to diploma & $50(21.5)$ \\
Grade 9 or below & \\
Maternal educational level, n(\%) & $84(36.1)$ \\
Bachelor degree or above & $109(46.8)$ \\
Grade 10 to diploma & $40(17.2)$ \\
Grade 9 or below & \\
Paternal occupation, n(\%) & $176(75.5)$ \\
Professional/Managerial & $57(24.5)$ \\
Others & \\
Maternal occupation, n(\%) & $179(76.8)$ \\
Professional/Managerial & $54(23.2)$ \\
Others & \\
Parental marital status, n(\%) & $220(94.4)$ \\
Married and living together & $13(5.6)$ \\
Divorced/separation/cohabited & $5.95(1.53)$ \\
WDQ externalizing problems (range: 0-20), mean(SD)
\end{tabular}

Table 2 Correlations among variables of interest

\begin{tabular}{|c|c|c|c|c|c|c|c|}
\hline \multicolumn{2}{|l|}{ Variable } & \multirow[t]{2}{*}{1} & \multirow[t]{2}{*}{2} & \multirow[t]{2}{*}{3} & \multirow[t]{2}{*}{4} & \multirow[t]{2}{*}{5} & \multirow[t]{2}{*}{6} \\
\hline 1 & Change in anxiety & & & & & & \\
\hline 2 & Change in stress & $0.500 * * *$ & & & & & \\
\hline 3 & Change in depression & $0.430 * * *$ & $0.595 * * *$ & & & & \\
\hline 4 & Change in adjustment problems & 0.021 & 0.088 & -0.010 & & & \\
\hline 5 & Change in parental stress & -0.065 & -0.041 & -0.081 & 0.088 & & \\
\hline 6 & $\begin{array}{l}\text { Adolescent family life satisfaction } \\
\text { (before COVID-19) }\end{array}$ & 0.094 & 0.125 & 0.055 & $0.137 *$ & 0.001 & \\
\hline Mean (SD) & & $0.13(5.42)$ & $0.12(7.32)$ & $0.84(6.22)$ & $-0.48(4.33)$ & $1.87(7.07)$ & $16.81(3.56)$ \\
\hline
\end{tabular}

${ }^{*} p<0.05 ; * * * p<0.001$

with parents, and initial level of externalizing problems and distress variable of interest. The second step in the analysis tested the hypothesis that adolescent T1 family life satisfaction would moderate a relationship between parental stress and adolescent psychological distress changes at T2. Significant interactions were found between adolescent T1 family life satisfaction and parental T2 elevated stress in predicting adolescent $\mathrm{T} 2$ changes in anxiety $(\beta=0.21$, $\mathrm{p}=0.034)$ and stress levels $(\beta=0.24, \mathrm{p}=0.014)$. To examine these interactions graphically, we plotted the regression of T2 changes in psychological distress (stress, depression, and anxiety) on T1 family life satisfaction at high and low levels for adolescents facing elevated parental stress and those who did not at T2 (see Fig. 2a-c). Compared to those low in family life satisfaction at T1, adolescents with higher levels of family life satisfaction at $\mathrm{T} 1$ reported increased levels of anxiety and stress when parental stress was elevated at T2, but their levels of anxiety and stress were reduced when 


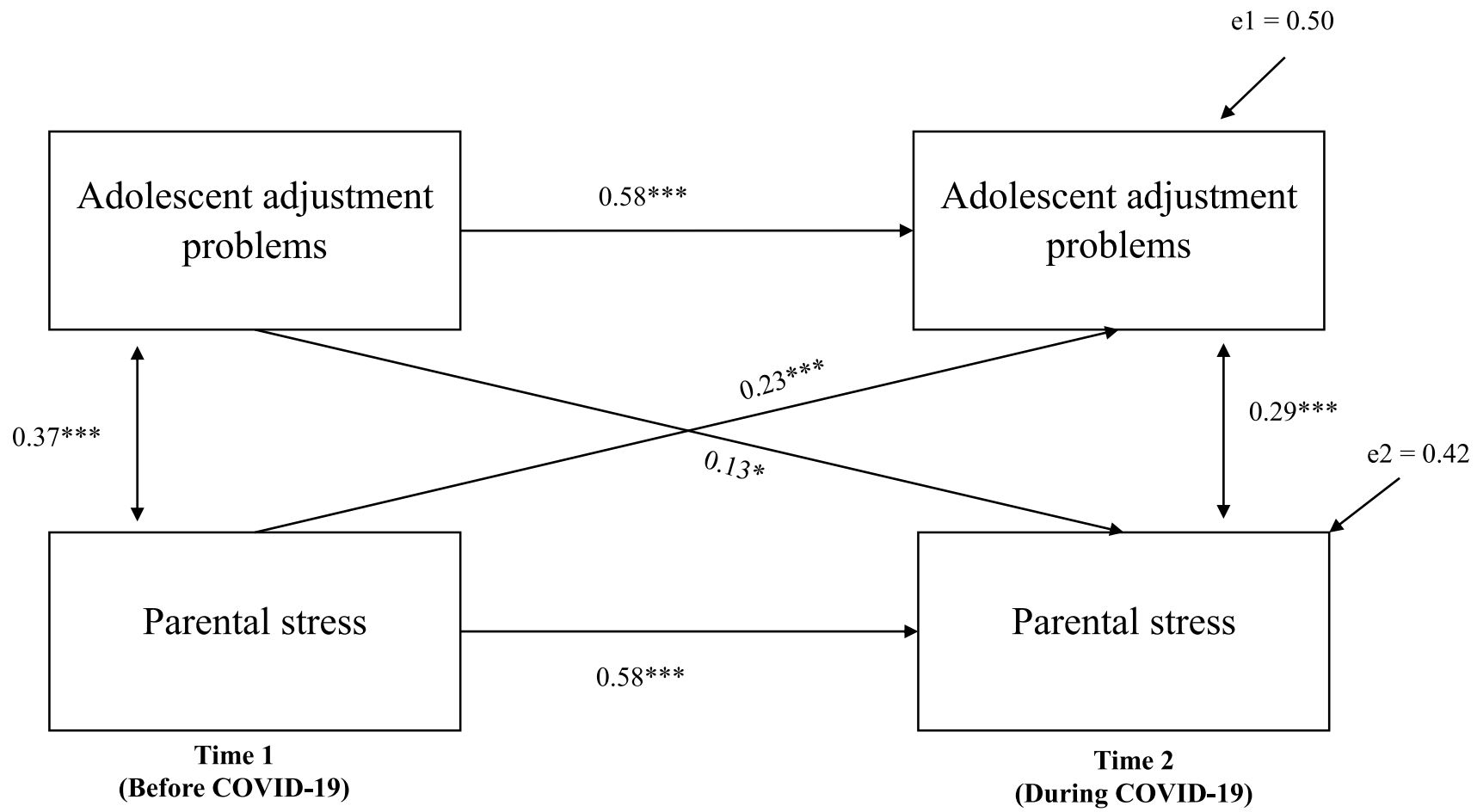

Fig. 1 Cross-lagged effects of adolescent adjustment problems and parental stress. Coefficients were standardized and a full information maximum likelihood estimator was used. $* \mathrm{p}<0.05 ; * * \mathrm{p}<0.01 ; * * * \mathrm{p}<0.001$

Table 3 Adolescent T1 family life satisfaction as a moderator of the association between changes in parental stress and adolescent psychological distress at $\mathrm{T} 2$

\begin{tabular}{|c|c|c|c|c|c|c|}
\hline & \multicolumn{2}{|c|}{$\begin{array}{l}\text { Mean change in } \\
\text { DASS anxiety }\end{array}$} & \multicolumn{2}{|c|}{$\begin{array}{l}\text { Mean change in } \\
\text { DASS stress }\end{array}$} & \multicolumn{2}{|c|}{$\begin{array}{l}\text { Mean change in } \\
\text { DASS depres- } \\
\text { sion }\end{array}$} \\
\hline & $\beta$ & p-value & $\beta$ & p-value & $\beta$ & p-value \\
\hline Change in parental stress & -0.11 & 0.152 & -0.14 & 0.061 & -0.08 & 0.301 \\
\hline $\begin{array}{l}\text { Adolescent perception of relationship with parents } \\
\text { (before COVID-19) }\end{array}$ & -0.17 & 0.053 & -0.18 & 0.042 & -0.21 & 0.033 \\
\hline Parental stress change $\mathrm{X}$ relationship with parents & 0.21 & 0.034 & 0.24 & 0.014 & 0.17 & 0.119 \\
\hline
\end{tabular}

Adjusted for age, gender, family SES, parental marital status, status of receiving financial assistance from the government, frequency of dinner with parents, and initial level of externalizing problems and distress variable of interest parental stress showed no increase at $\mathrm{T} 2$. On the other hand, T1 family life satisfaction did not play a moderating role in the relationship between changes in parental stress and changes in adolescent depression symptoms at T2. As shown in Fig. 2c, adolescents reported increased levels of depression when parental stress was elevated at $\mathrm{T} 2$ regardless of their initial level of family life satisfaction at $\mathrm{T} 1$.

\section{Discussion}

Despite a growing body of literature on stressors associated with the COVID-19 pandemic, only a few studies focused on early adolescents' pre-pandemic feelings or perception of family environment which is believed to be an important determinant of how one would react during and following natural disasters or other major sociohistorical events (Wilson et al., 2010). Our findings support a bidirectional model between parental stress and perception of early adolescents' adjustment problems in the pandemic context. Furthermore, we found that parental stress predicted a marked increase in levels of adolescent stress and anxiety over the course of the pandemic only for those adolescents high in pre-pandemic family life satisfaction. The findings together provide new insights into the family antecedents and correlates of psychosocial wellbeing among children in early adolescence during the COVID-19 pandemic. 
Fig. 2 Changes in adolescent psychological distress at low vs. high family life satisfaction for adolescents facing elevated parental stress vs. those who did not during COVID-19

\section{a) Anxiety}

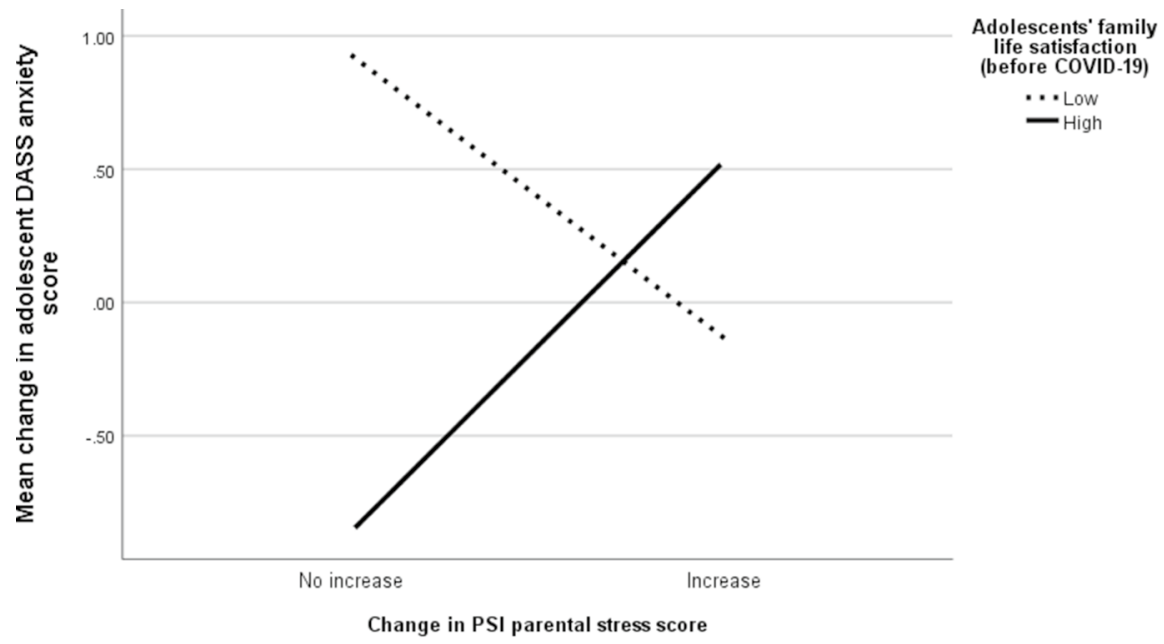

b) Stress

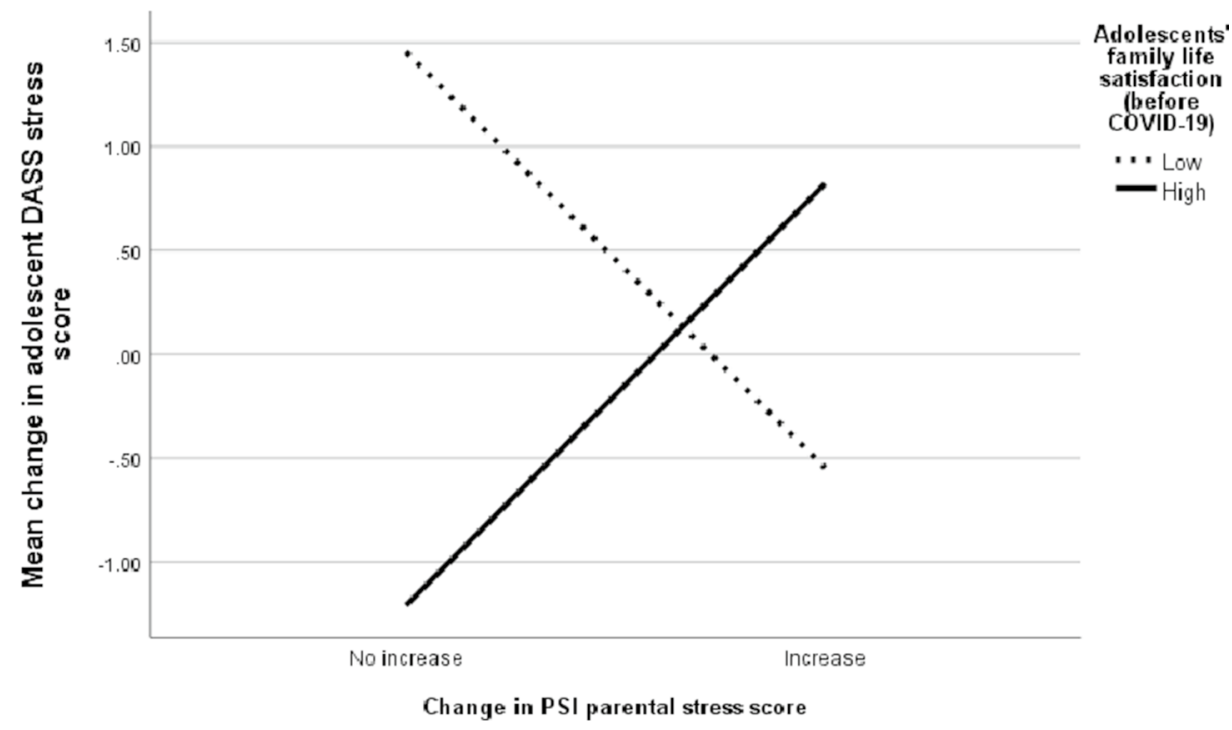

c) Depression

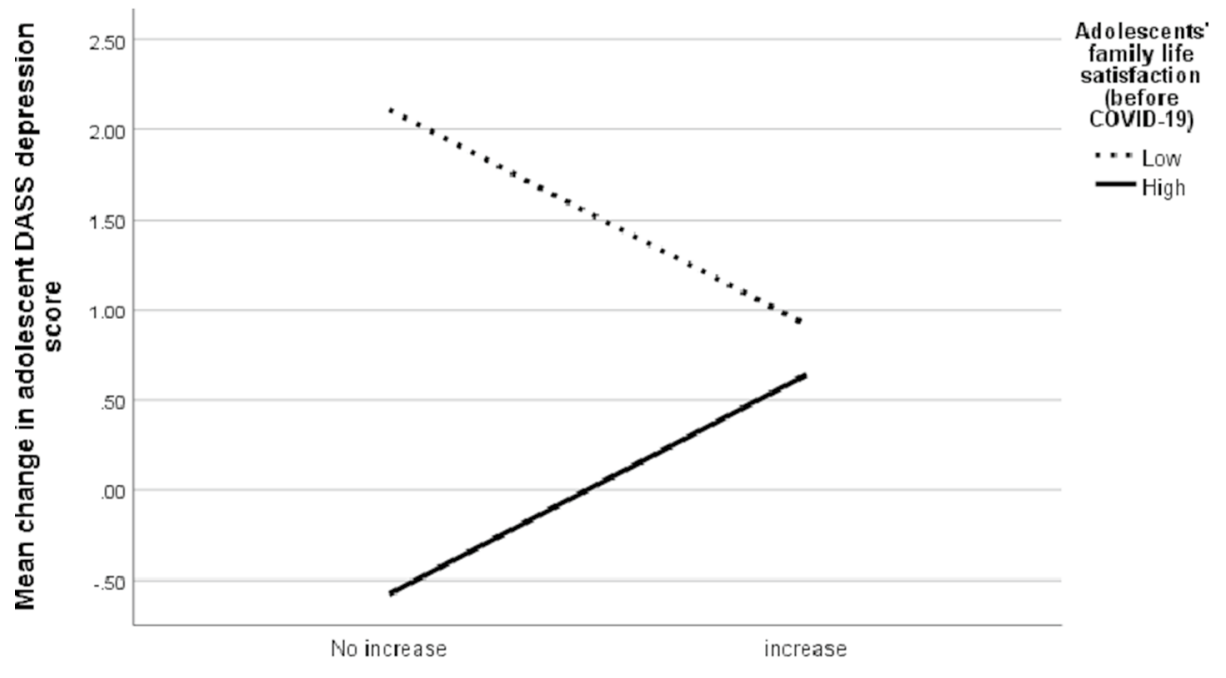

Change in PSI parental stress score 
Results of this study also align with previous non-pandemic research showing reciprocal influences of parental practices and child behavioral outcomes (Rodas et al., 2017). Although we did not investigate parenting practices, it is possible that parental perception of adolescent psychosocial difficulties before the pandemic could lead to inappropriate discipline practices and elevated stress levels during the pandemic. In line with the family systems perspectives (Nelson et al., 2009), our results suggest that parents without mental health problems before the pandemic may have a higher capacity to help children to cope with pandemic-related stressors. They are also less likely to transfer negative emotions to other members, and thus these families could face lower risks of poor health and well-being during the pandemic.

In addition, while the cross-lagged model provides evidence for the direction of the association between parental stress and their perception of adolescent adjustment problems, the interaction model supports the emotion spillover hypothesis within a family, particularly for those families having positive parent-adolescent interactions before the pandemic. Specifically, we observed that within these families, when parents experienced no increase in stress levels, pre-pandemic satisfaction with family life appeared to reduce adolescent negative feelings during the pandemic. However, when parental stress was elevated, pre-pandemic satisfaction with family life may predispose adolescents within these families to mental health problems during the pandemic. It is possible that elevated stressful experiences during the pandemic may have disrupted the usual family routine and interactions that are considered important for promoting adolescent perception of family life satisfaction before the pandemic. For example, when parents are stressed by pandemic-related challenges, they may show less interest to perform the usual pre-pandemic family activities or use harsher parenting methods which could aggravate emotional difficulties in children during the pandemic (Benner \& Mistry, 2020).

Compared to depressive symptoms, adolescent stress and anxiety symptoms were more likely to increase with parental stress during the COVID-19 pandemic, whereas the interactive effect of parental stress and family life satisfaction was larger for adolescent stress than for adolescent anxiety symptoms. These findings support the notion of stressor exposure as a risk factor for more severe mental health problems such as anxiety and depression (Anyan \& Hjemdal, 2016). As McEwen (2005) indicates, the challenges posed by the COVID-19 pandemic can evoke stress but not necessarily overwhelm individuals to have long-term health and functional problems such as chronic fatigue, worry, frustration or other coping difficulties. During the initial phase of the COVID-19 pandemic, with limited disruptions to family life and work, the surveyed parents and adolescents in this study may have undergone only the short-terms effects of the pandemic as indicated by greater increases in stress levels than the levels of anxiety and depression symptoms. However, as the COVID-19 pandemic continues to unfold, chronic exposure to adverse conditions could result in toxic stress responses and increase the risk of depression (Hammen, 2005). It is therefore important to conduct regular assessments, particularly on high-risk individuals, for early detection and intervention of unusual psychological and behavioural signs across different phases of the pandemic.

Despite the significant results, this study has several limitations. First, this study collected data at two time points only. Future studies should include three or more data collection time points which would increase the robustness of the findings by separating the between-person from the within-person effects (Berry \& Willoughby, 2017). Second, as indicated earlier, some of the mental health changes may take place at a slower rate and would not be adequately reflected in the initial phase of the COVID-19 pandemic. Third, our results are based on questionnaire assessment. Future research should use data from direct assessment or multiple informants' reports to increase the reliability of study findings. Finally, this study was not able to rule out the possibility of an intermediate variable that is responsible for the link between parental stress and adolescent adjustment problems. Future studies should measure a wider range of variables to elucidate such underlying mechanism.

\section{Conclusion}

This study provides evidence of significant family pre-pandemic influences on parental and adolescent mental health conditions during the pandemic. Our results add to the literature that mental health difficulties can spill over from one member to another member within the family during the pandemic. Prolonged home confinement can disminih pre-pandemic family life satisfaction on one hand and increase parental stress on the other hand, both of which may predispose children in early adolescence to mental health problems. More attention should thus be directed to those adolescents who undergo significant family life changes during the pandemic.

Acknowledgements We thank the schools, children, and parents for participation in this study. This study was supported by two grants from the Research Grants Council of the Hong Kong Special Administrative Region, China (General Research Fund (Project No. 17606318) and Collaborative Research Fund (Project No. C7009-19GF).

Author contributions Dr Rosa Wong contributed to this article including conceptualized the paper, analyzed the data, and wrote the initial draft. Mr Keith Tung conceptualized the paper, analyzed the data, and critically revised the manuscript. Dr. Shirley Li and Dr Celine Chui supervised data collection and analysis, and reviewed and revised the manuscript. Dr Winnie Tso, Prof. Tatia Lee, Prof. Ko Ling Chan, 
Prof. Yun K Wing, and Dr. King Wa Fu provided critical feedback and edits to subsequent revisions of the manuscript. Prof. Ian Wong and Dr Patrick Ip conceptualized and designed the study, coordinated and supervised data collection, and critically reviewed the manuscript. The corresponding authors attest that all listed authors meet authorship criteria and that no others meeting the criteria have been omitted. All authors approved the final manuscript as submitted and agree to be accountable for all aspects of the work.

Funding The study was supported by two grants from the Research Grants Council of the Hong Kong Special Administrative Region, China (General Research Fund (Project No. 17606318) and Collaborative Research Fund (Project No. C7009-19GF)).

Data availability The data that support the findings of this study are available on request from the corresponding author.

Code availability Not applicable.

\section{Declarations}

Financial Disclosure All authors have indicated they have no financial relationships relevant to this article to disclose.

\section{Clinical Trial Registration (if any) N/A}

Conflict of interest All authors have indicated they have no potential conflicts of interest to disclose.

\section{References}

Abidin, R. R. (1997). Parenting Stress Index: A measure of the parentchild system. In C. P. Zalaquett \& R. J. Wood (Eds.), Evaluating stress: A book of resources (pp. 277-291). Scarecrow Education.

Anyan, F., \& Hjemdal, O. (2016). Adolescent stress and symptoms of anxiety and depression: Resilience explains and differentiates the relationships. Journal of Affective Disorders, 203, 213-220. https://doi.org/10.1016/j.jad.2016.05.031

Barroso, N. E., Mendez, L., Graziano, P. A., \& Bagner, D. M. (2018). Parenting Stress through the Lens of Different Clinical Groups: A Systematic Review \& Meta-Analysis. Journal of Abnormal Child Psychology, 46(3), 449-461. https://doi.org/10.1007/ s10802-017-0313-6

Benner, A. D., \& Mistry, R. S. (2020). Child Development During the COVID-19 Pandemic Through a Life Course Theory Lens. Child Development Perspectives, 14(4), 236-243. https://doi.org/ 10.1111/cdep. 12387

Berry, D., \& Willoughby, M. T. (2017). On the practical interpretability of cross-lagged panel models: Rethinking a developmental workhorse. Child development, 88(4), 1186-1206. https://doi.org/ 10.1111/cdev.12660

Breaux, R. P., Harvey, E. A., \& Lugo-Candelas, C. I. (2016). The Role of Parent Psychopathology in Emotion Socialization. Journal of Abnormal Child Psychology, 44(4), 731-743. https://doi.org/10. 1007/s10802-015-0062-3

Buuren, S. v., \& Groothuis-Oudshoorn, K. (2010). mice: Multivariate imputation by chained equations in R. Journal of statistical software, 1-68.

Conger, R. D., Wallace, L. E., Sun, Y., Simons, R. L., McLoyd, V. C., \& Brody, G. H. (2002). Economic pressure in African American families: A replication and extension of the family stress model.
Developmental Psychology, 38(2), 179-193. https://doi.org/10. 1037/0012-1649.38.2.179

Elgar, F. J., Craig, W., \& Trites, S. J. (2013). Family Dinners, Communication, and Mental Health in Canadian Adolescents. Journal of Adolescent Health, 52(4), 433-438. https://doi.org/10. 1016/j.jadohealth.2012.07.012

Evans, S., Mikocka-Walus, A., Klas, A., Olive, L., Sciberras, E., Karantzas, G., \& Westrupp, E. M. (2020). From "It Has Stopped Our Lives" to "Spending More Time Together Has Strengthened Bonds": The Varied Experiences of Australian Families During COVID-19. Frontiers in Psychology, 11(2906). https://doi.org/ 10.3389/fpsyg.2020.588667

Fegert, J. M., Vitiello, B., Plener, P. L., \& Clemens, V. (2020). Challenges and burden of the Coronavirus 2019 (COVID-19) pandemic for child and adolescent mental health: A narrative review to highlight clinical and research needs in the acute phase and the long return to normality. Child and Adolescent Psychiatry and Mental Health, 14(1), 20. https://doi.org/10. 1186/s13034-020-00329-3

Goodman, R. (2001). Psychometric Properties of the Strengths and Difficulties Questionnaire. Journal of the American Academy of Child \& Adolescent Psychiatry, 40(11), 1337-1345. https://doi. org/10.1097/00004583-200111000-00015

Hammen, C. (2005). Stress and Depression. Annual Review of Clinical Psychology, 1(1), 293-319. https://doi.org/10.1146/annurev.clinp sy.1.102803.143938

HKSAR Census and Statistics Department. (2016). Hong Kong 2016 Population By-census - Thematic Report:Ethnic Minorities. Retrieved 18 December from https://www.censtatd.gov.hk/hkstat/ sub/sp459.jsp?productCode $=$ B1120100

Holmes, E. A., O'Connor, R. C., Perry, V. H., Tracey, I., Wessely, S., Arseneault, L., Ballard, C., Christensen, H., Cohen Silver, R., Everall, I., Ford, T., John, A., Kabir, T., King, K., Madan, I., Michie, S., Przybylski, A. K., Shafran, R., Sweeney, A., ... Bullmore, E. (2020). Multidisciplinary research priorities for the COVID-19 pandemic: A call for action for mental health science. The Lancet Psychiatry, 7(6), 547-560. https://doi.org/10.1016/ S2215-0366(20)30168-1

Hu, L. T., \& Bentler, P. M. (1999). Cutoff criteria for fit indexes in covariance structure analysis: Conventional criteria versus new alternatives. Structural equation modeling, 6(1), 1-55.

Huang, R., Ho, S., Wang, M., Lo, W., \& Lam, T. (2016). Reported alcohol drinking and mental health problems in Hong Kong Chinese adolescents. Drug and Alcohol Dependence, 164, 47-54.

Kline, R. B. (2015). Principles and practice of structural equation modeling. Guilford publications.

Lam, C. B., McHale, S. M., \& Crouter, A. C. (2012). Parent-child shared time from middle childhood to late adolescence: Developmental course and adjustment correlates. Child Development, 83(6), 2089-2103. https://doi.org/10.1111/j.1467-8624.2012. 01826.x

Lau, J. T. F., Yang, X., Tsui, H. Y., Pang, E., \& Wing, Y. K. (2006). Positive mental health-related impacts of the SARS epidemic on the general public in Hong Kong and their associations with other negative impacts. Journal of Infection, 53(2), 114-124. https://doi. org/10.1016/j.jinf.2005.10.019

Lee, J. (2020). Mental health effects of school closures during COVID19. The Lancet Child \& Adolescent Health, 4(6), 421. https://doi. org/10.1016/S2352-4642(20)30109-7

Liang, L., Yang, J., \& Yao, S. (2019). Measurement equivalence of the SDQ in Chinese Adolescents: A horizontal and longitudinal perspective. Journal of Affective Disorders, 257, 439-444.

Little, T. D., Preacher, K. J., Selig, J. P., \& Card, N. A. (2007). New developments in latent variable panel analyses of longitudinal data. International Journal of Behavioral Development, 31(4), 357-365. 
Liu, Z., Tang, H., Jin, Q., Wang, G., Yang, Z., Chen, H., Yan, H., Rao, W., \& Owens, J. (2020). Sleep of preschoolers during the coronavirus disease 2019 (COVID-19) outbreak. e13142. https://doi. org/10.1111/jsr.13142

McEwen, B. S. (2005). Stressed or stressed out: what is the difference? Journal of psychiatry \& neuroscience : JPN, 30(5), 315-318. https://pubmed.ncbi.nlm.nih.gov/16151535 https://www.ncbi. nlm.nih.gov/pmc/articles/PMC1197275/

Neece, C. L., Green, S. A., \& Baker, B. L. (2012). Parenting Stress and Child Behavior Problems: A Transactional Relationship Across Time. American Journal on Intellectual and Developmental Disabilities, 117(1), 48-66. https://doi.org/10.1352/1944-7558-117.1. 48

Nelson, J. A., O’Brien, M., Blankson, A. N., Calkins, S. D., \& Keane, S. P. (2009). Family stress and parental responses to children's negative emotions: Tests of the spillover, crossover, and compensatory hypotheses. Journal of Family Psychology, 23(5), 671-679. https://doi.org/10.1037/a0015977

Ni, M. Y., Yao, X. I., Leung, K. S. M., Yau, C., Leung, C. M. C., Lun, P., Flores, F. P., Chang, W. C., Cowling, B. J., \& Leung, G. M. (2020). Depression and post-traumatic stress during major social unrest in Hong Kong: A 10-year prospective cohort study. The Lancet, 395(10220), 273-284. https://doi.org/10.1016/S01406736(19)33160-5

Oosterhoff, B., Palmer, C. A., Wilson, J., \& Shook, N. (2020). Adolescents' Motivations to Engage in Social Distancing During the COVID-19 Pandemic: Associations With Mental and Social Health. Journal of Adolescent Health, 67(2), 179-185. https://doi. org/10.1016/j.jadohealth.2020.05.004

Pattusamy, M., \& Jacob, J. (2017). The Mediating Role of Familyto-Work Conflict and Work-Family Balance in the Relationship between Family Support and Family Satisfaction: A Three Path Mediation Approach. Current Psychology, 36(4), 812-822. https://doi.org/10.1007/s12144-016-9470-y

Prime, H., Wade, M., \& Browne, D. T. (2020). Risk and resilience in family well-being during the COVID-19 pandemic. American Psychologist, 75(5), 631-643.

R Core Team. (2013). R: A language and environment for statistical computing.

Resnick, M. D., Bearman, P. S., Blum, R. W., Bauman, K. E., Harris, K. M., Jones, J., Tabor, J., Beuhring, T., Sieving, R. E., \& Shew, M. (1997). Protecting adolescents from harm: Findings from the National Longitudinal Study on Adolescent Health. JAMA, 278(10), 823-832.

Rodas, N. V., Chavira, D. A., \& Baker, B. L. (2017). Emotion socialization and internalizing behavior problems in diverse youth: A bidirectional relationship across childhood. Research in Developmental Disabilities, 62, 15-25. https://doi.org/10.1016/j.ridd. 2017.01.010

Romero, E., López-Romero, L., Domínguez-Álvarez, B., Villar, P., \& Gómez-Fraguela, J. A. (2020). Testing the effects of COVID-19 confinement in Spanish children: The role of parents' distress, emotional problems and specific parenting. International Journal of Environmental Research \& Public Health, 17(19), 6975.

Rothbaum, F., \& Weisz, J. R. (1994). Parental caregiving and child externalizing behavior in nonclinical samples: A meta-analysis. Psychological Bulletin, 116(1), 55.
Schermerhorn, A. C., \& Mark Cummings, E. (2008). Transactional Family Dynamics: A New Framework for Conceptualizing Family Influence Processes. In R. V. Kail (Ed.), Advances in Child Development and Behavior (Vol. 36, pp. 187-250). JAI. https:// doi.org/10.1016/S0065-2407(08)00005-0

Tam, K. K., Chan, Y. C., \& Wong, C. K. M. (1994). Validation of the parenting stress index among Chinese mothers in Hong Kong. Journal of Community Psychology, 22(3), 211-223.

Taouk, M. M., Lovibond, P. F., \& Laube, R. (2001). Psychometric properties of a Chinese version of the short depression anxiety stress scales (DASS21). Cumberland hospital. Retrieved 14 December 2020 from http://www2.psy.unsw.edu.au/dass/Chine se/Chinese\%20DASS21\%20Paper.pdf

Tso, W. W. Y., Wong, R. S., Tung, K. T. S., Rao, N., Fu, K. W., Yam, J. C. S., Chua, G. T., Chen, E. Y. H., Lee, T. M. C., Chan, S. K. W., Wong, W. H. S., Xiong, X., Chui, C. S., Li, X., Wong, K., Leung, C., Tsang, S. K. M., Chan, G. C. F., Tam, P. K. H., ... lp, P. (2020). Vulnerability and resilience in children during the COVID-19 pandemic. European Child \& Adolescent Psychiatry. https://doi.org/10.1007/s00787-020-01680-8

Van Lancker, W., \& Parolin, Z. (2020). COVID-19, school closures, and child poverty: A social crisis in the making. The Lancet Public Health, 5(5), e243-e244. https://doi.org/10.1016/S2468-2667(20) 30084-0

Vyas, S., \& Kumaranayake, L. (2006). Constructing socio-economic status indices: How to use principal components analysis. Health Policy Planning, 21(6), 459-468.

Wilson, A. C., Lengua, L. J., Meltzoff, A. N., \& Smith, K. A. (2010). Parenting and temperament prior to September 11, 2001, and parenting specific to $9 / 11$ as predictors of children's posttraumatic stress symptoms following 9/11. Journal of Clinical Child and Adolescent Psychology, 39(4), 445-459. https://doi.org/10.1080/ 15374416.2010.486317

Wong, R. S., Tung, K. T. S., Rao, N., Ho, F. K. W., Chan, K. L., Fu, K.-W., Tso, W. W. Y., Jiang, F., Yam, J. C. S., Coghill, D., Wong, I. C. K., \& Ip, P. (2021). A Longitudinal Study of the Relation between Childhood Activities and Psychosocial Adjustment in Early Adolescence. 18(10), 5299. https://www.mdpi.com/1660$4601 / 18 / 10 / 5299$

$\mathrm{Wu}, \mathrm{Q} .$, \& Xu, Y. (2020). Parenting stress and risk of child maltreatment during the COVID-19 pandemic: A family stress theoryinformed perspective. Developmental Child Welfare, 2(3), 180196. https://doi.org/10.1177/2516103220967937

Xie, X., Xue, Q., Zhou, Y., Zhu, K., Liu, Q., Zhang, J., \& Song, R. (2020). Mental Health Status Among Children in Home Confinement During the Coronavirus Disease 2019 Outbreak in Hubei Province. China. JAMA Pediatrics, 174(9), 898-900. https://doi. org/10.1001/jamapediatrics.2020.1619

Publisher's note Springer Nature remains neutral with regard to jurisdictional claims in published maps and institutional affiliations. 